\title{
Política fiscal, asequibilidad y efectos cruzados de precios en la demanda de productos de tabaco: el caso de Uruguay
}

\author{
Alejandro Ramos Carbajales, MSc, ${ }^{(1)}$ Dardo Curti, Lic en Econ. ${ }^{(2)}$
}

\author{
Ramos-Carbajales A, Curti D. \\ Política fiscal, asequibilidad y efectos cruzados \\ de precios en la demanda de productos \\ de tabaco: el caso de Uruguay \\ Salud Publica Mex 2010;52 supl 2:S186-S196.
}

\section{Resumen}

Uruguay es un país que desde 2005 ha realizado una política activa de control del tabaco. Sin embargo, la evolución de la demanda del total de productos de tabaco muestra un descenso insignificante en los últimos cinco años, lo que es contrario a lo esperado. La hipótesis es que el fuerte crecimiento del ingreso de los hogares unido a una elasticidad-ingreso de la demanda de cigarrillos cercana a I fue uno de los factores que contrarrestó el aumento real en los precios vía impuestos. El aumento en el ingreso de los hogares fue de $36 \%$ en términos reales en el periodo 2005-2009 debido a la fuerte recuperación luego de la crisis del año 2002. Por otro lado, un segundo factor explicativo importante de la demanda de cigarrillos en el Uruguay es la sustituibilidad entre cigarrillos y tabaco de armar. El impuesto y precio del tabaco de armar sigue siendo sustancialmente más bajo que el del cigarrillo, de forma que en los últimos años la cantidad demandada de tabaco de armar ha subido. El trabajo consistió entonces en revisitar un estudio de demanda realizado en 2004 por los autores y volver a estimar una función demanda de los dos productos principales de tabaco en el Uruguay (cigarrillos y tabaco de armar), lo que permite estimar las elasticidades precio, ingreso y cruzadas. A partir de estas estimaciones se realiza un ejercicio de simular alternativas de incrementos de impuestos con lo que se evalúa qué aumentos son necesarios

\author{
Ramos-Carbajales A, Curti D. \\ Fiscal policy, affordability and cross effects \\ in the demand for tobacco products: \\ the case of Uruguay. \\ Salud Publica Mex 2010;52 suppl 2:SI86-SI 96.
}

\begin{abstract}
Uruguay, a country with a solid tobacco control policy since 2005 shows, contrary to expectations, an insignificant decrease in total tobacco products' sales in the last five years. The hypothesis is that on one side, changes in household income and the income elasticity of the demand for cigarettes were important countervailing factors in the demand of both products. The period 2005-2009 shows a large increase of $36 \%$ in household real income in Uruguay due to fast economic recovery after the 2002 crisis. The second factor is the interchangeability of roll your own and cigarettes and the impact on the demand of each product as a reaction to tax and price changes. The tax and price of roll your own tobacco remains substantially lower than that of cigarettes. This fact, and the increased substitution of roll your own for cigarettes seems to be the main reasons for the low impact of the policy of tobacco tax and price increases. This paper then consists of a revision of a 2004 study to estimate separate demands for both main tobacco products and obtain estimates for own price, cross price and income elasticities. Then, a simulation study was performed using the elasticities found and two scenarios of increases in household income: moderate $(2.5 \%$ per year) and high ( $5 \%$ per year) confirming that countries where income is growing fast and with a potential for substitution towards cheaper products require
\end{abstract}

(I) Centro de Investigación para la Epidemia del Tabaquismo (CIET). Montevideo, Uruguay.

(2) Banco Central del Uruguay. Montevideo, Uruguay.

Fecha de recibido: 26 de marzo de 2010 - Fecha de aceptado: 13 de julio de 2010 Solicitud de sobretiros:Alejandro Ramos. Centro de Investigación para la Epidemia del Tabaquismo (CIET). Carlos Ma. Maggiolo 469 Apto 60I, Montevideo, I I 300 Uruguay. Correo electrónico: aleram22@gmail.com 
para realmente impactar sobre la demanda en un escenario de crecimiento del ingreso de los hogares moderado de $2.5 \%$ anual y alto de $5 \%$ anual. Se confirma que se necesitan aumentos de impuestos muy superiores a los verificados en el último quinquenio.

Palabras clave: política fiscal para reducir el consumo de productos de tabaco; aumentos de precios e impuestos de productos de tabaco; demanda de cigarrillos y tabaco de armar en Uruguay substantial cigarette tax and price increases for a fiscal tobacco control policy to become effective.

Key words: fiscal policy to reduce the demand for tobacco products; tobacco tax and price increases; demand for cigarettes and hand rolled tobacco in Uruguay
$E^{n}$ Uruguay se fuman cigarrillos y tabaco de armar. a prevalencia global siete días de tabaquismo en Uruguay para mayores de 15 años fue estimada en $24.8 \%$ en 2008 (29.4\% hombres y $20.9 \%$ mujeres) a partir de ENPTA, * una encuesta nacional en poblaciones de 2500 y más habitantes.

Si bien el consumo de cigarrillos es predominante en la población fumadora, el tabaco de armar ha ido incrementando su consumo hasta alcanzar el $20 \%$ del total. Los jóvenes y las personas de menor ingreso son habitualmente los consumidores de tabaco de armar.

Uruguay es un país $100 \%$ libre de humo de tabaco en ambientes laborales y espacios públicos cerrados desde mayo de 2006 (Decreto Presidencial 268/05); en febrero de 2008 se aprobó la Ley 18.256 de Control del Tabaco con disposiciones respecto a la prohibición de todo tipo de publicidad y promoción en la venta de cigarrillos, extensas advertencias sanitarias con pictogramas, prohibiciones respecto de ventas a menores y multas a los infractores sobre espacios libres de humo.

Estudios ${ }^{1}$ realizados por los autores de este trabajo en 2004 habían permitido por primera vez un análisis científico de la demanda de productos de tabaco en Uruguay y otros países del Mercosur.

Dichos estudios econométricos determinaron para Uruguay una elasticidad-precio de la demanda de cigarrillos de -0.49 y del tabaco de armar de -0.88 , y una elasticidad-ingreso de 0.65 y -0.82 respectivamente.

En este contexto, y luego de transcurridos cinco años del estudio anterior, los autores realizaron un estudio actualizado ${ }^{\ddagger}$ de demanda para revisar los análisis anteriores y corroborar si las estimaciones realizadas en 2004 se mantenían o divergían por otros elementos

\footnotetext{
* Encuesta Nacional de Prevalencia del Tabaquismo (ENPTA), Centro de Investigación para la Epidemia del Tabaquismo, (CIET Uruguay), 2008

* Ramos A, Curti D. Uruguay: Análisis de la evolución reciente de la política de impuestos y precios sobre los productos de tabaco (2005-2009). CIET, Working paper; diciembre 2009.
}

que hubieran podido intervenir como explicativos de la demanda en estos últimos años.

Cabe destacar que los resultados de las estimaciones de 2004 estaban alineados con los de múltiples otros trabajos en diversos países desarrollados y en países de ingreso medio.

El periodo desde 2005 interesa además porque presentó en Uruguay un fuerte aumento en el ingreso de los hogares. Entre 2005 y 2009 existió un aumento de $36 \%$ en el ingreso medio de los hogares en términos reales debido a la recuperación económica luego de la crisis del año 2002. Incluso el año 2009, de recesión importante en las economías más desarrolladas y en varios países de la región, en Uruguay sólo se redujo la tasa de crecimiento del PIB a 2.9\%, desde un promedio superior a $6 \%$ en los cinco años anteriores.

En este periodo, si bien se plantearon aumentos de impuestos a los productos de tabaco, se mantuvieron tasas y bases impositivas menores para el tabaco de armar que para los cigarrillos. Esto ostensiblemente mantenía condiciones favorables al cambio de productos.

\section{Antecedentes en el Uruguay}

\section{Mercado}

El mercado de cigarrillos (industriales y a partir de tabaco de armar) se estima en 3.6 miles de millones de unidades anuales.*

En el país operan tres empresas tabacaleras, dos multinacionales (Phillip Morris y BAT) y una nacional (Compañía Industrial de Tabacos Montepaz SA). Esta última tiene más del 70\% del mercado legal. El contrabando se ha estimado en un $18 \%$ de los cigarrillos; ${ }^{2}$ no

\footnotetext{
* Este cálculo se realiza a partir de la estimación del Instituto Nacional de Estadística (INE) de la población mayor de 15 años (2.6 millones), una prevalencia en mayores de 15 años de $24.8 \%$ y un consumo diario de 15.5 cigarrillos (industriales y provenientes del armado, ambos datos provenientes de la Encuesta ENPTA, CIET 2008).
} 
hay contrabando del tabaco de armar, el que representa un 20\% del consumo de productos de tabaco. La mayor parte del tabaco hoja se importa y dos de las empresas realizan el procesamiento secundario (Philip Morris y Montepaz).

\section{Sistema de imposición a los cigarrillos en el Uruguay}

Impuesto Específico Interno (Imesi)

En el Uruguay el tabaco está gravado con un impuesto a su consumo específico: el Impuesto Especifico Interno (Imesi), Tabaco; existe este tipo de impuesto también para la primera venta de combustibles y alcohol, bebidas con y sin alcohol, vehículos automotores, perfumería, consumos suntuarios, etc.

Desde julio de 2007 el Imesi es un impuesto específico (de suma fija por paquete) en el caso de los cigarrillos pero es ad valorem en el caso del tabaco de armar, con una base impositiva ficta menor que el precio de mercado. Su tasa actual es de $70 \%$ aplicable sobre un precio de cálculo que fija el Poder Ejecutivo. Dicho precio es cercano pero menor que el precio al consumidor, por tanto la tasa efectiva del impuesto respecto del precio de venta al consumidor es menor que la tasa nominal del impuesto.

La diferencia de tasas del impuesto sobre los cigarrillos y el tabaco de armar viene desde la década de los setenta. Al final del periodo de análisis de este estudio (2008) la tasa del Imesi de los cigarrillos era $68 \%$ y la del tabaco de armar 28 por ciento.

En julio de 2009 las tasas se unificaron en 70\% pero la base impositiva del paquete de tabaco de armar continuó siendo sustancialmente menor que la de los cigarrillos, lo que además se traducía en un precio al consumidor también mucho menor.

\section{Impuesto al Valor Agregado (IVA)}

El Impuesto al Valor Agregado (IVA) grava a la tasa básica del 22\% a los productos de tabaco desde julio de 2007.

La Reforma fiscal de dicho año extendió el IVA a diferentes productos, entre ellos los cigarrillos y demás productos del tabaco. Anteriormente si bien estaban legalmente incluidos dentro de los productos gravables, el Poder Ejecutivo los había excluido en la práctica al fijar la tasa en 0 por ciento.*

* Esta decisión probablemente se relacionó con el tradicional "lobby" de la industria de que una mayor imposición a los cigarrillos traería consigo caída en la recaudación o aumento del contrabando, o ambas.
La carga fiscal global (Imesi e IVA) de la marca de cigarrillos más vendida era en 2008 de $66 \%$ del precio al consumidor, con lo que resultaba de las más altas del continente. En el caso del tabaco de armar era del 41 por ciento.

\section{Evolución reciente del precio y la demanda de productos de tabaco en el mercado interno}

Desde marzo de 2005 se ha instaurado una política de control del tabaco que incluyó un componente de aumento de impuestos. Sin embargo, los resultados no son los esperables porque ha existido un tratamiento fiscal diferente al tabaco de armar y a los cigarrillos.

Esta política de diferenciar el tratamiento fiscal del tabaco de armar en realidad no fue propia del gobierno actual, sino que viene desde la década de los setenta y fue mantenida por todos los gobiernos anteriores.

Para evaluar en forma comparada el tabaco de armar y los cigarrillos industriales se confeccionó un índice de volumen físico global de venta de productos de tabaco tomando el peso promedio de un cigarrillo de armar y el de un cigarrillo industrial (figura 1).* Se observa que no ha existido una reduccion sensible del volumen de productos de tabaco vendidos en el Uruguay en los cinco últimos años. Asimismo, el volumen total de ventas en peso de los productos de tabaco presentó una muy leve caída desde 1991, ${ }^{\ddagger}$ el periodo considerado para el análisis econométrico de la demanda.

\section{Demanda}

Este resultado oculta una evolución opuesta entre las ventas de cigarrillos y las de tabaco de armar. Existe una clara evolución favorable al tabaco de armar, de una participación en el total de productos de tabaco de $27 \%$ a principios de los noventa a más de $35 \%$ en la etapa actual. Estos porcentajes se calcularon sobre la venta de tabaco legal (figura 2).

\footnotetext{
* Alos efectos de la conversión entre cigarrillos armados manualmente a partir de tabaco y cigarrillos industriales se tomó una muestra de cigarrillos de armar y se pesaron con una balanza de precisión. Este simple estudio determinó un peso promedio de $0.562 \mathrm{~g}$ en 26 observaciones, con una desviación típica de 0.1357 . Dado un paquete de $45 \mathrm{~g}$ de peso, se arman 80 cigarrillos en promedio, lo que indica que un paquete de armar en Uruguay contiene el equivalente a 4 paquetes de 20 cigarrillos industriales.

₹ Se dispuso de información de volumen de ventas internas de cigarrillos y de tabaco de armar desde 1991 hasta diciembre de 2008. Fuente: Dirección General Impositiva (DGI).
} 


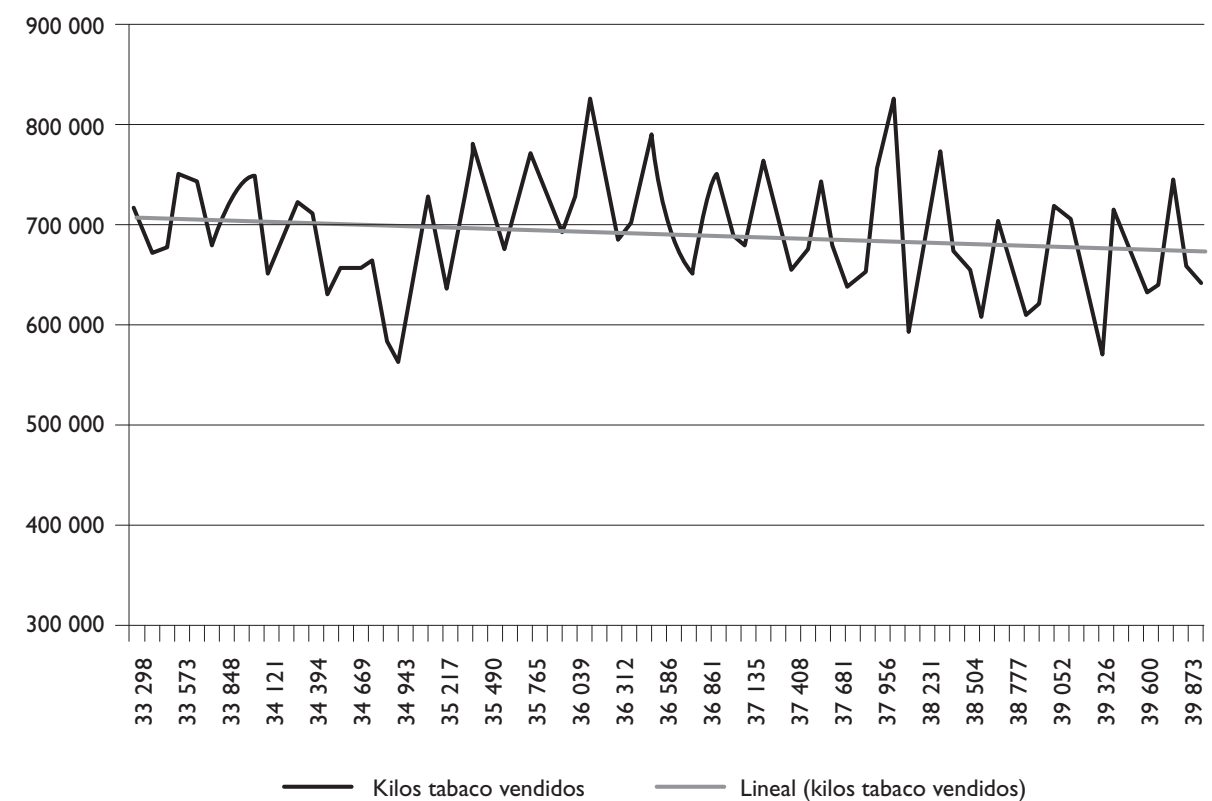

Fuente: Elaborado con datos de la Dirección General Impositiva (DGI)

Figura I.Volumen físico en Kilogramos de Ventas internas de tabaco de armar y CigarRillos. URUguay, I99 I-2008

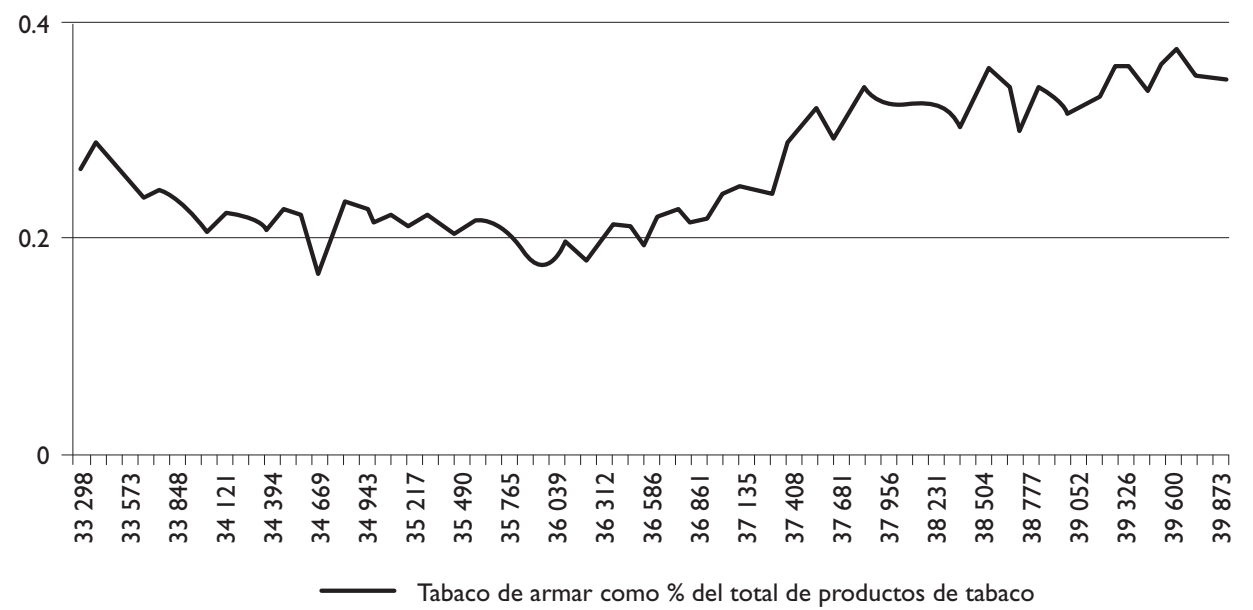

Fuente: Elaborado con datos de la Dirección General Impositiva (DGI) y estimaciones propias

Figura 2. Evolución del PORCENTAJe de VENTAS INTERNAS del VOLUMEN DE TABACO DE ARMAR SOBRE TOTAL DE VENTAS DE TODOS LOS PRODUCTOS DE TABACO. URUGUAY, I 99 |-2008

\section{Precios}

Los precios de los cigarrillos y del tabaco de armar en términos reales [o sea, deflactados por el Índice de precios del consumidor (IPC) del Instituto Nacional de Estadística (INE)] han evolucionado de modo similar y en los últimos trimestres el precio del tabaco de armar se ha reajustado hasta alcanzar el mismo nivel que el precio de los cigarrillos, por lo tanto el precio relativo de ambos productos no ha sufrido fuertes cambios en los últimos trimestres.

En el caso del tabaco de armar (figura 3) la cantidad vendida no disminuye a pesar del aumento en el precio, sino que permanece estable en los valores alcanzados 


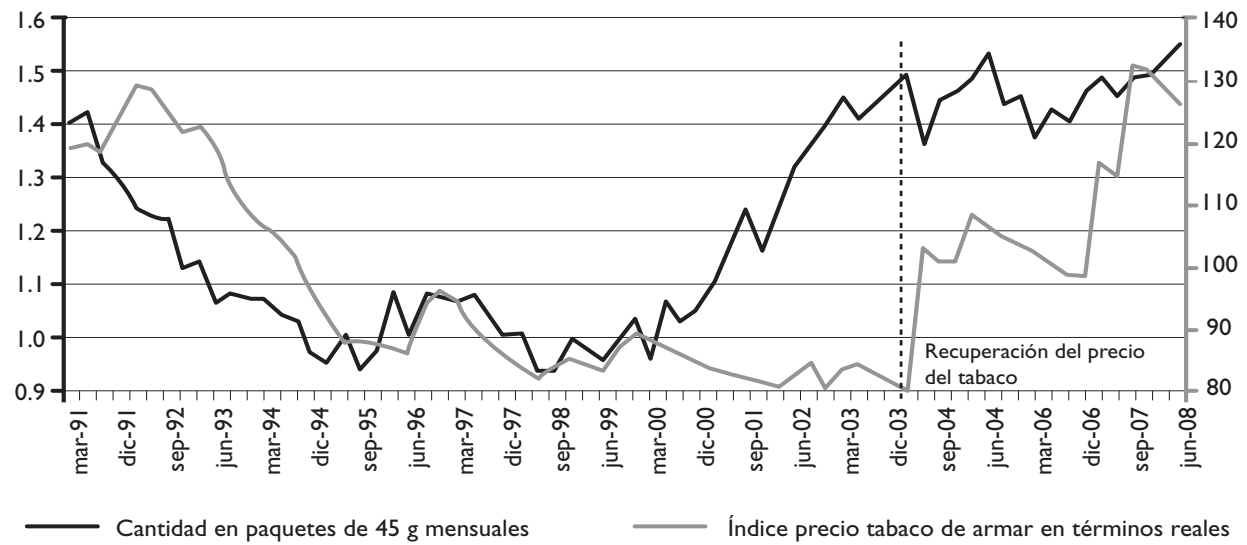

Fuente: Elaborado con datos de la Dirección General Impositiva (DGI) y del Instituto Nacional de Estadística (INE)

Figura 3.Ventas y precio del mercado interno del tabaco de armar. Uruguay, I 99 I-2008

desde 2003 e incluso se puede observar un leve aumento desde 2005 en adelante.

Este comportamiento de la cantidad vendida de tabaco de armar sugiere que algunos grupos de fumadores en los estratos socioeconómicos más bajos o los que fuman ambos productos pueden haber realizado un proceso de sustitución de cigarrillos por tabaco de armar; sin embargo su reacción no se puede explicar por el precio relativo entre un producto y otro, pues el mismo, como ya se comentó, no se modificó en el periodo observado.

Para el precio de los cigarrillos y la cantidad vendida desde 1998 se pueden identificar tres subperiodos (figura 4):

a) primer subperiodo en que la recesión (que comenzó en el primer trimestre de 1999 y terminó en el segundo trimestre de 2003) provocó la caída de las ventas en un porcentaje de $27 \%$, en tanto que el precio se mantuvo en valores en torno a 100 (el precio de los cigarrillos es un número índice).

b) El segundo subperiodo está dentro de la recuperación que la economía tiene desde el segundo trimestre de 2003 y las empresas tabacaleras inician un proceso de recuperación del precio de los cigarrillos buscando recomponer márgenes de beneficios. Este comportamiento de las empresas con el precio determinó que la cantidad vendida per cápita continuara cayendo (alrededor de un $10 \%$ ).

c) El último subperiodo se inicia en el primer semestre de 2007, al comenzar a aplicarse la política de aumento de los impuestos a los cigarrillos en el marco del convenio marco de control de tabaco firmado y ratificado por Uruguay. En este último subperiodo se verifica un aumento de $16 \%$ en términos reales en julio de 2007 y de $20 \%$ en junio de 2009 (no aparece en la figura).

Ingreso de los hogares

En el caso de los cigarrillos, la demanda evoluciona en igual sentido que el ingreso de los hogares, es decir que es un bien normal (figura 5).

Desde 2003 en adelante el efecto ingreso es dominante para la demanda de tabaco de armar (figura 6), y a pesar del encarecimiento en términos reales de este producto la cantidad consumida no disminuyó.

Existen dos factores que operan en sentido contrario para que la demanda de tabaco de armar no haya disminuido sino aumentado; ellos son el aumento en el ingreso de los consumidores y el encarecimiento de los cigarrillos. El tabaco de armar es un sustituto cercano de los cigarrillos para consumidores con ciertas características. ${ }^{*}$

\section{La demanda de cigarrillos en función de la asequibilidad}

La capacidad de compra en función del ingreso y los precios es un indicador fundamental para medir entre otras cosas el éxito de las políticas fiscales de control del tabaco. Este indicador se denomina "asequibilidad" y

* Datos de la Encuesta ENPTA señalan que 18.1\% de los fumadores/ as son solamente de tabaco de armar, $75 \%$ de cigarrillos y el resto de ambos productos. 


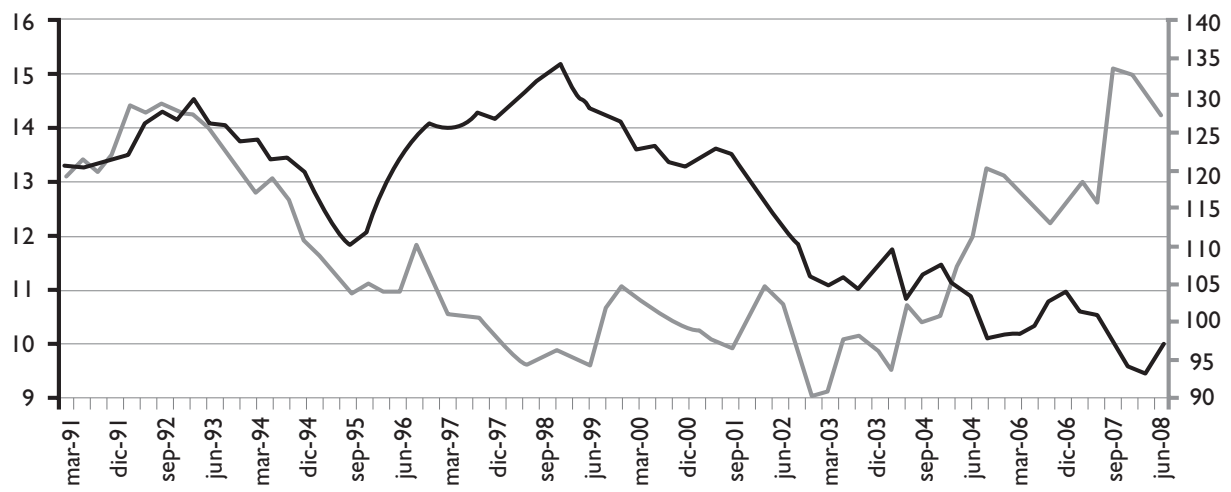

Cantidad en paquetes de cigarrillos mensualees _ Índice precios cigarrillos en términos reales (eje der.)

Fuente: Elaborado con datos de la Dirección General Impositiva (DGI) y del Instituto Nacional de Estadística (INE)

Figura 4.VENTAS y PRECIO MERCADO INTERNo de LOS CIGARRILLOS. URUGUAY, I 99 I-2008

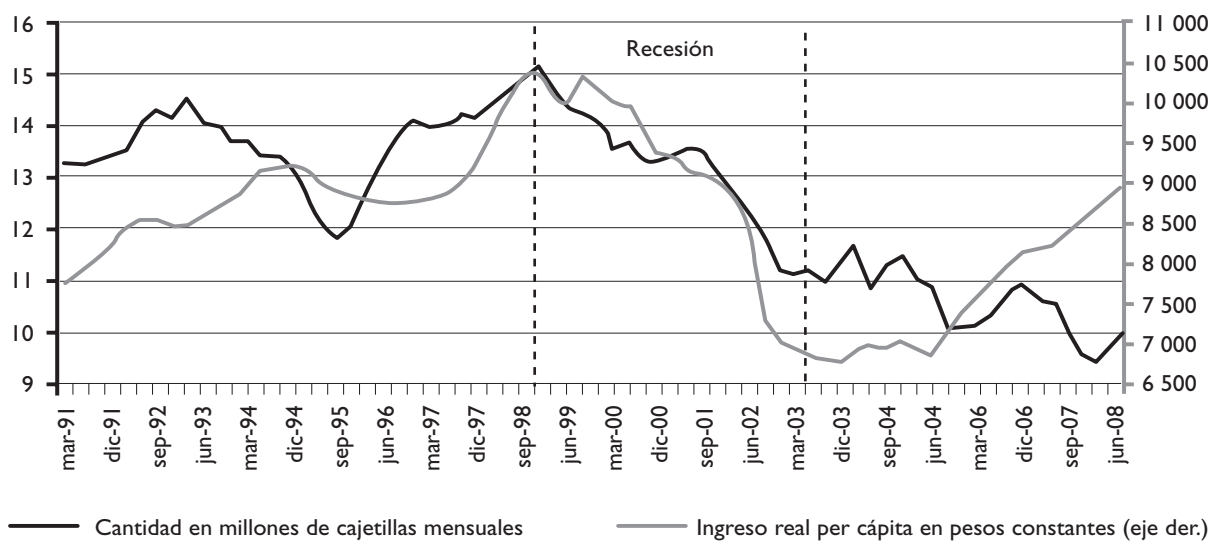

Figura 5. Evolución de ventas de cigarrillos e ingreso real. URuguay, I 99 I-2008

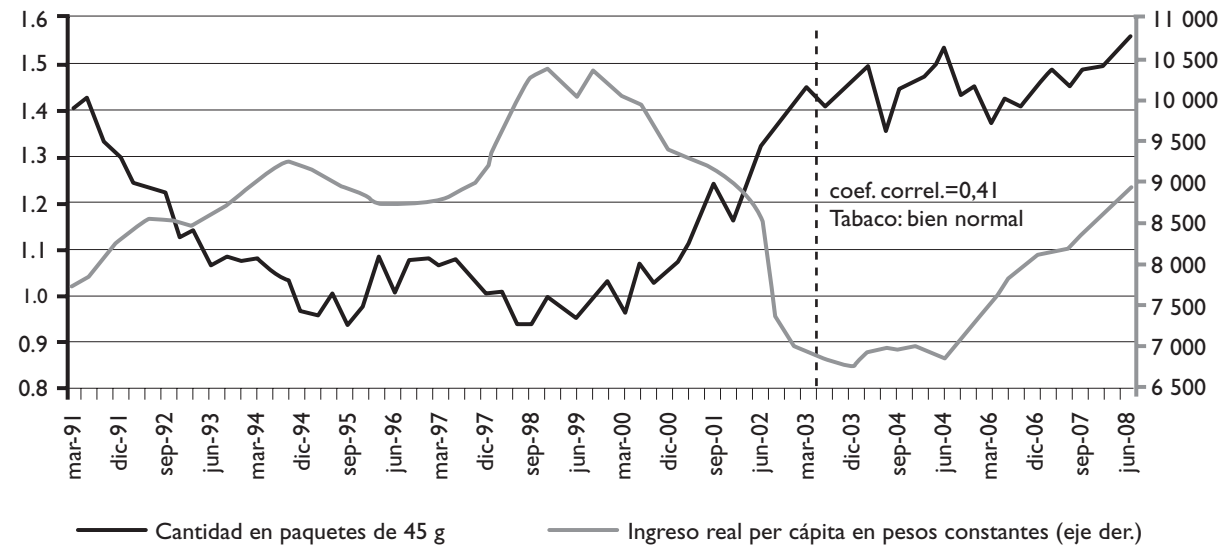

Fuente: Elaborado con datos de la Dirección General Impositiva (DGI) y del Instituto Nacional de Estadística (INE)

Figura 6. Evolución de ventas de tabaco de armar e ingreso Real. URuguay, I 99 |-2008 
mide la interacción entre los niveles de consumo de los fumadores según los distintos productos de tabaco, el ingreso y los precios que pagan por los mismos. ${ }^{3}$

$\mathrm{El}$ ingreso puede medirse de distintas formas. Una de ellas, que facilita las comparaciones entre países, es tomar el Producto Interno Bruto (PIB) per cápita. El indicador PIB / índice de precios cigarrillos, se denomina RIP (relative income price).

En el caso de Uruguay, para este estudio se usó el ingreso de los hogares como una medida más adecuada que el PIB y con ventajas dado su carácter más general que el ingreso por hora laboral. El indicador de asequibilidad utilizado es entonces el cociente del índice de evolución del ingreso de los hogares respecto del índice de precios de los cigarrillos. Si en un periodo el índice crece los cigarrillos se hacen más asequibles y viceversa.

Se observa (figura 7) que en el caso del cigarrillo, como del tabaco de armar, desde marzo de 1991 existen tres subperiodos diferenciados.

El primero hasta fines de 1997, en que la asequibiidad aumenta llegando a casi duplicarse.

Un segundo periodo, también sin politica de control de tabaco, entre 1998 y 2004, donde la asequibilidad disminuye fuertemente hasta alcanzar los niveles de inicios de los noventa. En este periodo ocurre un proceso recesivo de la economia uruguaya con fuerte caída del PIB en 2002 y recuperación que se produce en 2004.

Interesa en particular analizar el periodo más reciente, desde marzo de 2005. Se observa que si bien ha disminuido la asequibilidad de los cigarrillos y el tabaco de armar en el Uruguay, igualmente la situación no ha mejorado con respecto a la base de 1991, aunque sí con respecto al periodo inmediatamente anterior, entre los años 1998-2004.

Sin embargo, la situación del cigarrillo y del tabaco de armar es diferente.

A pesar del aumento en el ingreso de los hogares la cantidad demandada de cigarrillos continúa cayendo (figura 5) en el periodo de 2005 hasta 2008.

A este resultado puede haber coadyuvado que algunos grupos de consumidores hayan modificado su demanda, trasladándola en parte o totalmente hacia el consumo de tabaco de armar y que otro grupo de fumadores haya disminuido su consumo de cigarrillos* por el efecto de las otras medidas de control del tabaco (prohibición de fumar en lugares públicos desde marzo 2006 y prohibición de la publicidad desde 2008).

Incluso, como se verá en el análisis de los resultados, la elasticidad-ingreso del tabaco de armar es negativa, por lo que se contraponen dos efectos: 1 ) un aumento de la demanda de cigarrillos de fumadores de tabaco de armar por el incremento de su asequibilidad; 2) una tendencia que viene desde la época de la crisis

* Estos resultados sugieren que el análisis debería profundizarse a niveles más desagregados, para lo que se requiere información de encuestas y para varios periodos que permitan identificar el comportamiento temporal de los diversos grupos de consumidores.

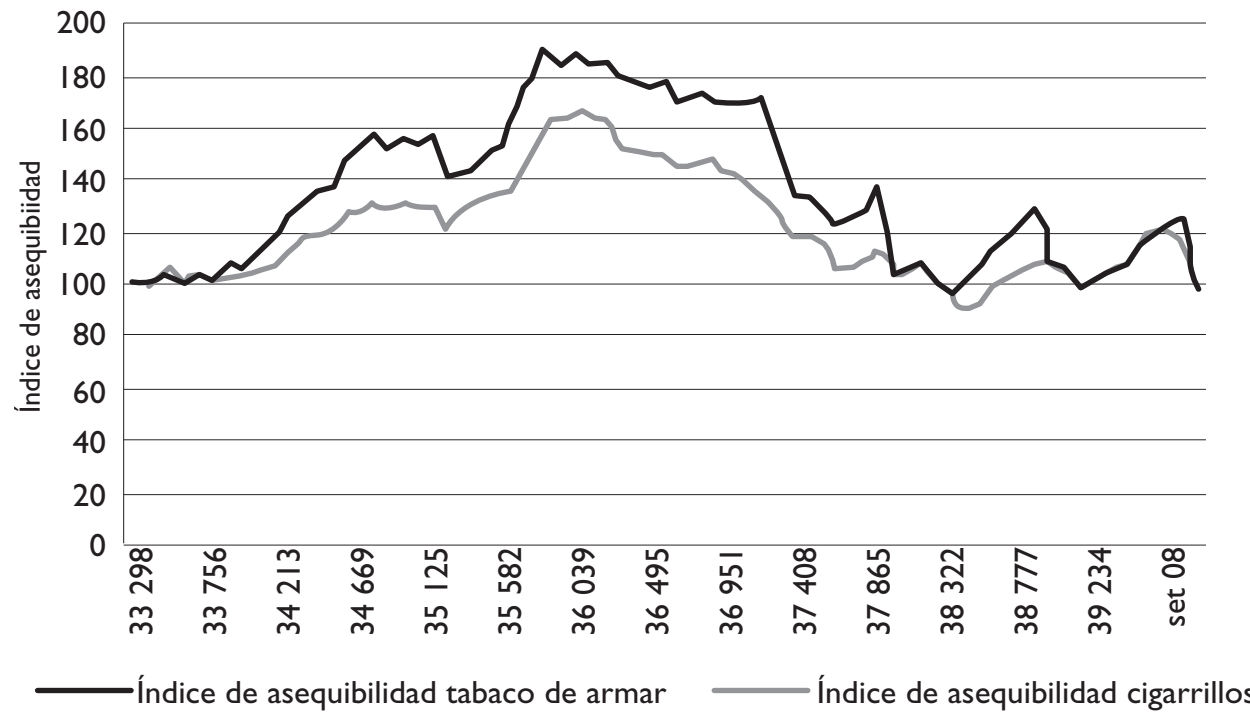

Fuente: Elaborado con datos de la Dirección General Impositiva (DGI) y del Instituto Nacional de Estadística (INE)

Figura 7. Asequibilidad de cigarillos y tabaco de armar en el Uruguay, I99 |-2008 
de 2002 de aumentar el consumo de tabaco de armar (cambio de gustos, preferencias, etc.)

Si bien el precio real del cigarrillo y del tabaco de armar aumentó desde 2005 como se observó, resulta necesario evaluar el efecto conjunto, sobre la demanda, del impacto de los precios de ambos productos; del aumento del ingreso de los hogares, y de una variable de tendencia para analizar la contribución de cada una de éstas.

\section{Análisis econométrico: los modelos de corrección de error y de ajuste parcial de la demanda de cigarrillos y tabaco de armar}

Se estimaron funciones de demanda de los productos de tabaco que se comercializan en Uruguay en forma legal, cigarrillos y tabaco de armar, para el periodo 1991 a 2008, con datos trimestrales.

Se trabajó con una función para los cigarrillos y otra para el tabaco de armar y se estimó un modelo de ajuste parcial y otro de corrección de error para cada una de aquellas funciones.

Se propone un modelo como el que sigue:*

$$
\operatorname{Ln} Q_{t}=b_{0}+b_{1} \operatorname{Ln} P_{t}+b_{2} \operatorname{Ln} Y_{t}+b_{3} T_{t}+b_{4} \operatorname{LnPOB} B_{t}+\sum_{j} b_{5 j} \operatorname{Ln} Q_{t_{t} j}+\varepsilon_{t}
$$

Qt es la cantidad demandada en $t$, Pt el precio del producto de tabaco en $\mathrm{t}$, Yt el ingreso de los consumidores en $\mathrm{t}$, Tt una tendencia determinística y POBt es el precio de otros bienes en el período t. Las variables precio, ingreso y cantidad demandada se expresan en logaritmos, de modo que los coeficientes asociados son las elasticidades de corto plazo.

En un modelo de corrección de errores la especificación se divide en la relación de largo plazo entre las variables, lo que supone una relación de equilibrio a largo plazo entre las mismas como la siguiente:

$$
\operatorname{Ln} Q_{t}=b_{0}+b_{1} \operatorname{Ln} P_{t}+b_{2} \operatorname{Ln} Y_{t}+b_{3} T_{t}+b_{4} \operatorname{LnPOB} B_{t}+\varepsilon_{t}
$$

Luego se especifica una relación de corto plazo, la que refleja el ajuste que hacen las diferentes variables para acercarse a la relación de largo plazo que existe entre ellas. La especificación es del tipo siguiente:

$d\left(\operatorname{Ln} Q_{t}\right)=b_{1} d\left(\operatorname{Ln} P_{t}\right)+b_{2} d\left(\operatorname{Ln} Y_{t}\right)+b_{4} d\left(\operatorname{LnPOB}_{t}\right)+(\alpha-1)\left(\right.$ residuos $\left._{t-1}\right)+\varepsilon_{t}(4)^{\ddagger}$

\footnotetext{
* Base de datos disponible a solicitud: Dardo Curti (dardocurti@ gmail.com).

₹ El operador de diferencias es $d$, de modo tal que por ejemplo $\left(\operatorname{LnQ}_{t}\right)=$ $\operatorname{LnQ}_{\mathrm{t}}-\operatorname{LnQ}_{\mathrm{t}-1}$
}

Siendo residuos ${ }_{t-1}=L N Q_{t-1}{ }^{-} b_{1} \operatorname{Ln} P_{t-1}+b_{2} \operatorname{Ln} Y_{t-1}+b_{3} T_{t-1}+b_{4} \operatorname{LnPOB} B_{t-1}$

El coeficiente 1- $\alpha$ representa la velocidad de ajuste hacia el equilibrio de largo plazo existente entre las variables.

Cabe señalar que Uruguay se destaca por sus políticas de control de tabaco en otras áreas, entre ellas, que fue el primer país de América Latina en prohibir fumar en espacios públicos cerrados y lugares de trabajo el 1 de marzo de 2006.

En las primeras estimaciones se incluyó una variable ficticia para modelar este cambio, pero ésta no fue significativa, por lo que se le excluyó luego. Este resultado puede deberse al corto periodo de vigencia incluido en el análisis.

\section{Resultados}

\section{Estimaciones para la demanda de cigarrillos}

La estimación de la función de demanda de cigarrillos da unas estimaciones de la elasticidad-precio directa que son -0.51 y -0.34 a corto plazo y -0.57 y -0.52 a largo plazo para el modelo de ajuste parcial y el modelo de corrección de error, respectivamente.

Estos resultados son similares a los obtenidos en el trabajo anterior citado de $2004^{*}$ y no revelan un cambio significativo en la elasticidad a pesar de que entre 2003 y 2008 el precio de los cigarrillos en términos reales aumentó 45 por ciento.

La elasticidad-ingreso para los cigarrillos se estimó en 0.42 y 0.68 a corto plazo y 0.47 y 1.02 a largo plazo para el modelo de ajuste parcial y el modelo de corrección de error, respectivamente. A pesar de las diferencias, las estimaciones no son estadísticamente diferentes (los intervalos de confianza se solapan).

La elasticidad-precio cruzada para los cigarrillos se estimó en 0.13 y 0.26 a corto plazo y 0.15 y 0.38 a largo plazo para el modelo de ajuste parcial y el modelo de corrección de error, respectivamente. Estos resultados tampoco permiten rechazar la hipótesis de que las estimaciones no son diferentes.

En suma, los resultados obtenidos para la demanda de cigarrillos son similares en cualquiera de los dos modelos estimados y muy similares a los obtenidos en el trabajo de 2003, lo que da robustez a las estimaciones efectuadas.

\footnotetext{
* La elasticidad-precio de la demanda en el estudio de 2004 fue -0.55 , con una desviación estándar de 0.06 , elasticidad-ingreso 0.72 , con desviación estándar 0.02 .
} 


\section{Resultados de las estimaciones para la demanda de tabaco de armar}

La estimación de la elasticidad-precio directa es de -0.18 y -0.37 a corto plazo y -0.24 y -0.44 a largo plazo para el modelo de ajuste parcial y el modelo de corrección de error, respectivamente. Estos resultados son bastante diferentes si se los compara con los obtenidos en la estimación realizada por los autores en $2004,{ }^{*}$ lo que sugiere estimaciones no robustas e inestabilidad en el parámetro de la variable precio del tabaco de armar.

La estimación de la elasticidad-precio cruzada fue de 1.18 y 0.59 a corto plazo y 1.53 y 0.69 a largo plazo en el modelo de ajuste parcial y en el modelo de corrección de error, respectivamente. Las estimaciones del modelo de ajuste parcial son notoriamente mayores a las obtenidas en el trabajo de 2003. Sin embargo los resultados del modelo de corrección de error no son muy diferentes a los obtenidos en el trabajo previo. Por último, la estimación de la elasticidad-ingreso da -0.85 y -1.07 a corto plazo y -1.11 y -1.24 a largo plazo en el modelo de ajuste parcial y en el modelo de corrección de error, respectivamente. Estas estimaciones son muy parecidas a las obtenidas en 2003.

En síntesis, las estimaciones de la elasticidadingreso y precio cruzada dan con los signos esperados y son similares a las obtenidas en el trabajo anterior. ${ }^{\ddagger}$

Sin embargo, la estimación de la elasticidad-precio directa en el caso del modelo de ajuste parcial da más baja en valor absoluto (en el modelo de corrección de error $-0.37 \mathrm{y}-0.44$ a corto y largo plazo, respectivamente) si se la compara con la obtenida en el trabajo de 2004 (-0.86 y -1.09 a corto y largo plazo, respectivamente).

Esta debilidad de los resultados para la estimación de la función de demanda de tabaco se puede explicar por los fuertes cambios que ha habido en las cantidades demandadas tanto de tabaco de armar y cigarrillos como en las tres variables que las explican en los modelos ensayados.

Se puede ver (figura 6) el cambio de la correlación fuerte negativa entre ingreso y cantidad demandada de tabaco de armar verificada hasta 2003 y desde esa fecha al presente la correlación positiva entre ingreso y cantidad.

\footnotetext{
* Elasticidad-precio propio -1.09, elasticidad ingreso -1.01 (nótese que este valor negativo indica que el tabaco de armar fue un bien inferior en el periodo de análisis anterior)

\# Es de destacar que si se elaboran intervalos de confianza para las estimaciones puntuales anteriores, éstos se solapan, por lo que no se puede rechazar la hipótesis de que las estimaciones no son diferentes.
}

En función de estos cambios se puede explicar que en las primeras estimaciones se obtuvo un resultado en el cual el precio no fue significativo en el modelo de corrección de error.

La alternativa que se utilizó fue entonces modelar el cambio estructural verificado desde 2003.

\section{Cambio estructural en la demanda de tabaco de armar desde el inicio de 2003}

El principal cambio entre la especificación inicial que daba no significativa la función de demanda de tabaco de armar es que se omitió modelar el cambio estructural en el primer trimestre de 2003 [2003(1T)].

Las siguientes pruebas muestran precisamente la existencia de dicho cambio estructural.

Prueba de Chow de cambio estructural en 2003(IT)

\begin{tabular}{lccc}
\multicolumn{5}{c}{ Chow breakpoint test: 2003 Q I } \\
F-statistic & 2.894895 & Prob. F(7,53) & 0.012257 \\
\hline Log likelihood ratio & 21.69334 & Prob. Chi-Square(7) & 0.002869
\end{tabular}

Este test se aplica sobre el modelo original, en el que no se incluye la variable que modela la existencia de cambio estructural. La hipótesis nula de la prueba de Chow es que no hay cambio estructural en 2003(1T), sin embargo los valores p llevan a rechazar tal hipótesis, por lo que si se rechaza la hipótesis nula hay evidencia (al $5 \%$ de significación) de que hay cambio estructural.

Prueba de Ramsey (Reset test)

\begin{tabular}{lccc}
\multicolumn{4}{c}{ Ramsey } \\
RESET Test: & \\
F-statistic & 2.45679 I & Prob. F(2,58) & 0.094585 \\
\hline Log likelihood ratio & 5.448378 & Prob. Chi-Square(2) & 0.065599
\end{tabular}

La prueba de Ramsey comprueba entre otras cosas la posibilidad de variable omitida, incorrecta forma funcional, errores de medida que generan correlación entre los errores y las variables omitidas.

En este caso se aplica la primera posibilidad, es decir de que exista una variable omitida, pues no se modela el cambio estructural en el modelo originalmente propuesto para la función de demanda de tabaco de armar.

Esta prueba también se aplica sobre el modelo sin incluir la variable que modela el cambio estructural.

La hipótesis nula es que no hay una variable omitida (el modelo está bien especificado), y se rechaza la hipótesis nula al $10 \%$ de significación. 


\section{Prueba de la razón de verosimilitud}

\begin{tabular}{lccc}
\multicolumn{4}{c}{ Omitted variables: DTABA } \\
F-statistic & 13.56865 & Prob. F(I,59) & 0.000501 \\
\hline Log likelihood ratio & 13.86870 & Prob. Chi-Square(I) & 0.000196
\end{tabular}

La prueba de la razón de verosimilitud verifica si existe una variable omitida en la especificación; en este caso se prueba con la variable DTABA, que es una tendencia que se inicia en 2003(1T). La hipótesis nula de la prueba es que la variable mencionada está bien omitirla, es decir que supone a priori que la variable no es significativa. El resultado de los valores p lleva a rechazar la hipótesis anterior (al $1 \%$ de significación), por lo que se valida incluir la variable de tendencia DTABA.

\section{En sintesis:}

Las pruebas anteriores son evidencia de que hay un cambio estructural desde 2003 en adelante en la función de demanda de tabaco de armar. Por tanto, en los modelos que se presentan se incluyó una variable de tendencia desde 2003(1T) hasta el fin del periodo. Esto eliminó el problema del coeficiente de la variable precio en las estimaciones del parámetro del precio en el modelo de corrección de error.

Este cambio puede explicarse a partir de la fuerte crisis económica del país en 2002 con fuerte caída del producto y del ingreso de los hogares. Una nueva generación de fumadores comenzó a volcarse progresivamente al tabaco de armar, producto que tradicionalmente era más barato porque el Imesi tenía desde mucho tiempo atrás una tasa de $27 \%$ frente al $68 \%$ de los cigarrillos.

Puede entonces aducirse que el cambio en las preferencias y gustos venía siendo motivado por los precios relativos de los productos, pero era muy progresivo y lento en periodos anteriores y se agudizó a partir de la crisis, confirmando un cambio de preferencias de parte de los fumadores, particularmente los jóvenes. * O sea, el aumento del ingreso de los hogares ya no los volcó como antes a abandonar el tabaco de armar y pasarse a los cigarrillos.

El tabaco de armar, de ser un bien inferior pasó a ser un bien normal luego de 2003.

\footnotetext{
* Esto se observó en la encuesta ENPTA de 2008.
}

\section{Simulaciones de incremento de precios e ingresos}

Este ejercicio trata de mostrar con ayuda del paquete econométrico Eviews cuál es el efecto combinado de las elasticidades precio propio, cruzadas, ingreso y la tendencia (que también fue incluida en estas simulaciones, lógicamente) frente a distintas alternativas de aumento de impuestos y escenarios respecto del aumento del ingreso de los hogares.

Se analizaron dos escenarios para estas simulaciones en función del posible aumento del ingreso de los hogares en un periodo de 12 meses. ${ }^{*}$ En el periodo comprendido entre comienzos de 2005 y fines de 2009 el ingreso de los hogares subió $36 \%$ en términos reales, indicando una media acumulada de más de $6 \%$ anual.

Por tanto, en el escenario 1 se plantearon aumentos del ingreso de los hogares de $2.5 \%$ anual y en el escenario 2 se plantearon aumentos de $5 \%$ anual.

Dentro de cada escenario se plantearon distintas hipótesis de aumentos de precios de los cigarrillos y del tabaco de armar. En cada caso, detrás de los aumentos de precios, tomando como fijo el precio del industrial y los márgenes de comercialización, el aumento depende del Imesi. ${ }^{\ddagger}$

En el primer escenario se proyecta un aumento del ingreso de $2.5 \%$ en el año, un aumento del precio del tabaco de armar de $40 \%$ y alternativamente, aumentos de los precios de los cigarrillos entre 20 y 40 por ciento.

Los resultados muestran que aun cuando los cigarrillos aumentaran $20 \%$ en el precio (lo que requeriría un aumento del Imesi de 25\%), aun con un escenario moderado de incremento del ingreso de los hogares, si el tabaco de armar aumenta $40 \%$ la demanda de cigarrillos no sólo no caerá, sino que subirá $1.4 \%$ al cabo del año.

A medida que los aumentos de precios de los cigarrillos se van haciendo mayores se produce el efecto buscado de reducción de la demanda. Pero aun con $40 \%$ de incremento de precios de ambos productos la demanda caería en este escenario 1 solamente alrededor de $6 \%$ para ambos productos.

En el segundo escenario se duplican todos los aumentos para analizar cómo impactan aumentos de

\footnotetext{
* El resultado es ilustrativo, ya que correspondería simular varios años de incremento de ingresos de los hogares.

₹ Para obtener un determinado aumento del precio final al consumidor se requiere un aumento porcentual del Imesi de alrededor del doble en el caso del tabaco de armar y de alrededor de $25 \%$ más en el caso de los cigarrillos.
} 
precios en un entorno donde también sube el ingreso de los hogares en forma importante $(5 \%$ en un periodo de 12 meses).

Se observa que aumentando un $80 \%$ el precio del tabaco de armar si los cigarrillos aumentan sólo 40\% la reducción del consumo de cigarrillos es sumamente baja y estimada en 1.3 por ciento. En el otro extremo, si los cigarrillos y el tabaco de armar sufren aumentos de precios de $80 \%$ (para lo que requieren $100 \%$ de aumento del Imesi en el caso de los cigarrillos y $160 \%$ en el caso del tabaco de armar) las reducciones del consumo de ambos productos, sobre todo del tabaco de armar, siguen siendo muy modestas.

Este ejercicio de simulación demuestra que la política fiscal sobre los cigarrillos y el tabaco de armar en el Uruguay, en un escenario de aumento importante del ingreso de los hogares, debe manejar incrementos considerables del Imesi para tener impacto sobre el consumo, que es el resultado sanitario buscado.

\section{Limitaciones del análisis}

Las funciones de demanda en las estimaciones realizadas no incluyen variables no económicas del control del tabaco que el Uruguay implementó a partir de 2006. Esto es en parte consecuencia de que las medidas, como la prohibición de fumar en espacios públicos y lugares de trabajo cerrados, fueron efectivas durante un corto lapso en el periodo de análisis.

El periodo de análisis desde 1991 incluye un cambio estructural en 2003(1T). Esto se comprobó y se modeló con la inclusión de una variable de tendencia determinística. Una alternativa hubiera sido considerar solamente para el estudio el periodo posterior a 2003(1T). Sin embargo, dado que el número de trimestres hubiera sido insuficiente para realizar las estimaciones, esto queda como ejercicio para un estudio posterior.

\section{Conclusiones. Lecciones de política}

En los países como Uruguay, donde se consume más de un producto de tabaco, las políticas de aumentos de impuestos deben tomar en cuenta las elasticidades cruzadas de los productos sustitutos y la presión fiscal existente en el punto de partida. Los impuestos se deben aumentar de modo que los precios relativos no favorez- can claramente un producto sobre otro, como ha sido el caso de Uruguay desde hace muchos años. Si esto no se toma en cuenta se corre el riesgo de que los aumentos de impuestos puedan generar caídas de demanda de uno de los productos (en este caso, los cigarrillos) pero incrementos en la del otro producto (en este caso, el tabaco de armar) aun cuando su precio absoluto pueda haber aumentado. Y esto puede implicar que no se reduzca el consumo global de productos de tabaco, que es el objetivo de la política sanitaria.

Como el caso de Uruguay lo enseña, en periodos de tres a cinco años un país puede tener aumentos del ingreso del orden del 30\% o más, lo que con elasticidades ingreso cercanas o superiores a 1 implica que la demanda de productos de tabaco aumente también 30\% por este motivo. Esto hace necesario que una política de control de tabaco que utilice adecuadamente la poderosa herramienta del aumento de impuestos realice, ex ante, proyecciones de la evolución del ingreso y que periódicamente se analice un aumento de los impuestos de los productos de tabaco de forma que no sólo no aumente, sino que disminuya la asequibilidad de estos productos para los consumidores.

\section{Declaración de conflicto de intereses}

Declaramos no tener conflicto de intereses.

\section{Agradecimientos}

Los autores agradecen al financiamiento del IDRC de Canadá al Centro de Investigación para la Epidemia del Tabaquismo (CIET Uruguay) que hizo posible este estudio.

\section{Referencias}

I. Ramos A, Curti D.WHO. Economics of tobacco control in the countries of MERCOSUR (Argentina, Brazil, Paraguay, Uruguay, Chile, Bolivia). LUGAR:World Health Organization, 2005. www.who.int/entity/ tobacco/research/economics/publications/mercorsur_studies/en/ 2. Centro de Investigación para la Epidemia del Tabaquismo (CIET Uruguay). Encuesta Nacional de Prevalencia de Tabaquismo (ENPTA). LUGAR: CIET Uruguay, 2008

3. Blecher $\mathrm{E}$, van Walbeek C.An analysis of cigarette affordability. Paris: International Union Against Tuberculosis and Lung Disease, 2008. 